\title{
Modeling Uncertainty of Induced Technological Change
}

\author{
Andrii Gritsevskyi \\ Nebojša Nakićenović
}

RR-00-24

November 2000

Reprinted from Energy Policy, 28 (2000) 907-921.

International Institute for Applied Systems Analysis - Schlossplatz 1 - A-2361 Laxenburg - Austria Tel: (+43 2236) 807 • Fax: (+43 2236) 71313 • E-mail: publications@iiasa.ac.at •Web: www.iiasa.ac.at 
Research Reports, which record research conducted at IIASA, are independently reviewed before publication. Views or opinions expressed herein do not necessarily represent those of the Institute, its National Member Organizations, or other organizations supporting the work.

Reprinted from Energy Policy 28 (2000) 907-921

Copyright (c) 2000, with permission from Elsevier Science Ltd.

All rights reserved. No part of this publication may be reproduced or transmitted in any form or by any means, electronic or mechanical, including photocopy, recording, or any information storage or retrieval system, without permission in writing from the copyright holder. 


\title{
Modeling uncertainty of induced technological change
}

\author{
Andrii Gritsevskyi, Nebojša Nakićenović* \\ Environmentally Compatible Energy Strategies Project, IIASA A-2361 Laxenburg, Austria \\ Received 16 May 2000
}

\section{Abstract}

This paper presents a new method for modeling-induced technological learning and uncertainty in energy systems. Three related features are introduced simultaneously: (1) increasing returns to scale for the costs of new technologies; (2) clusters of linked technologies that induce learning depending on their technological "proximity" in addition to the technology relations through the structure (and connections) of the energy system; and (3) uncertain costs of all technologies and energy sources.

The energy systems-engineering model MESSAGE developed at IIASA was modified to include these three new features. MESSAGE is a linear programming optimization model. The starting point for this new approach was a global (single-region) energy systems version of the MESSAGE model that includes more than 100 different energy extraction, conversion, transport, distribution and end-use technologies. A new feature is that the future costs of all technologies are uncertain and assumed to be distributed according to the log-normal distribution. These are stylized distribution functions that indirectly reflect the cost distributions of energy technologies in the future based on the analysis of the IIASA energy technology inventory. In addition, the expected value of these cost distributions is assumed to decrease and variance to narrow with the increasing application of new technologies. This means that the process of technological learning is uncertain even as cumulative experience increases. New technologies include, for example, fuel cells, photovoltaic and wind energy conversion technologies.

The technologies are related through the structure of energy system in MESSAGE. For example, cheaper wind energy has direct and indirect effects on other technologies that produce electricity up-stream and on electric end-use technologies downstream. In addition, technologies are grouped into clusters that depend on technological "proximity." For example, the costs of all fuel cells for mobile applications are a function of their combined installed capacity weighted according to their expected unit sizes. This relationship depends on how closely the technologies are related. This varying degree of "collective" technological learning for technologies belonging to the same cluster is also uncertain.

Each scenario of alternative future developments for a deterministic version of the global energy systems model MESSAGE requires approximately $10 \mathrm{~min}$ of CPU time on PC with Intel Pentium II $233 \mathrm{MHz}$ and $128 \mathrm{MB}$ of RAM. Therefore, it is simply infeasible to generate alternative future developments under uncertainty based on a simple Monte-Carlo type of analysis were one sequentially draws observations from the very large number of more than 200,000 cost distributions (100 technologies, 11 time steps, 10 technological clusters with 22 technologies included) assumed here for modeling technological learning and uncertainty. Instead, the new approach proposed here starts with a large but finite number of alternative energy systems "technology dynamics" and generates in "parallel" another large but finite number of deterministic scenarios by sampling from the distributions simultaneously for each of these technology dynamics. In this application, about 130,000 scenarios were generated. There were 520 alternative technology dynamics each with about 250 alternative deterministic scenarios resulting from the simultaneous stochastic samplings. Both numbers were initially varied before deciding that about 500 is a sufficient number of different technology dynamics required for a wide spectrum of alternative technological learning possibilities and that about 250 different deterministic scenarios is a sufficient number to generate most of the interesting future energy systems structures for each of the technology dynamics based on the analysis that in total produced roughly one million different scenarios. These large numbers of scenarios represent a very small subset of all possible ones that is basically infinite. They were not chosen randomly, but are a result of applying adaptive global search technique to the formulated non-convex, non-smooth stochastic problem.

From the 520 alternative technology dynamics, about 53 resulted in scenarios with very similar overall energy systems cost. They have fundamentally different technological dynamics and produce a wide range of different emergent energy systems but can be considered to be approximately equivalent with respect to "optimality" criteria (in this case simultaneous cost and risk minimization). Thus, one of the results of the analysis is that different structures of energy system emerge with similar overall costs, i.e., that there is a large diversity across alternative energy technology strategies. The strategies are path dependent and it is not possible to choose a priori "optimal" direction of energy systems development.

\footnotetext{
* Corresponding author. Tel.: 0043-2236-715-210; fax: 0043-2236-713-13.

E-mail addresses: gritsev@iiasa.ac.at (A. Gritsevskyi), naki@iiasa.ac.at (N. Nakićenović).
} 
Another result of the analysis is that the endogenous technology learning with uncertainty and spillover effects have the greatest impact on the emerging structures of energy system during the first few decades of the next century. Over these "intermediate" periods of time these two processes create effective lock-in effects and increasing returns to adoption. In the very long run, however, all of these effects are not of a great importance. The reason is that over such long periods many doubling of capacity of all technologies with inherent leaning occur so that there are few relative cost advantages that result from large investments in some technologies and clusters. Therefore, the main finding is that under uncertainty the near-term investment decisions in new technologies are more important in determining the direction of long-term development of the energy system than are the decisions that are made later, towards the end of the time horizon. Thus, the most dynamic phase in the development of future energy systems occurs during the next few decades. It is during this period that there is a high freedom of choice across future technologies and many of these choices lead to high spillover learning effects for related technologies.

One policy implication that can be made based on the emerging dynamics and different directions of energy systems development in this analysis is that future RD\&D efforts and investments in new technologies should be distributed across "related" technologics rather than be directed at only one technology from the cluster even if it appears as a "winner." Another implication is that it is better not to spread RD\&D efforts and technology investments across a large portfolio of future technologies. It is rather better to focus on (related) technologies that might form technology clusters. (C) 2000 Elsevier Science Ltd. All rights reserved.

\section{Introduction}

Fundamental changes in global energy systems are slow. The substitution of traditional energy sources, e.g. wood, by coal with the advent of steam, steel and railways took most of the last century. The replacement of coal by oil and gas and associated technologies lasted the better part of this century. In contrast to these very slow processes of change, other parts of the energy system can be more dynamic, especially the evolution of end-use technologies. However, the fact that fundamental changes occur on the scale of 100 years rather than a few years mean that technological changes that have inherently shorter time constants need to be consistent with the overall, slower processes of change in the energy system. Thus, many generations of individual technologies that are replaced through the normal rate of capital turnover are a part of the overall slow change from older to newer sources of energy and other related structural changes in energy systems. This means that also in the future, it is likely that many generations of new technologies will come and go before the possible transition to the post-fossil era or to new-fossil systems is achieved. Therefore, there is an infinite number of alternative scenarios that lead to all possible future energy systems. The directions of these future transitions are clearly also uncertain. Future energy systems could rely on renewable energy sources, on clean coal, on less carbon-intensive fossils such as natural gas, or on nuclear power.

As was mentioned, replacement of primary energy sources has lasted for the better part of the last and the current century and implies that similar changes are conceivable during the next century. Climate change is characterized by long time constants just as energy systems are. It might take a few decades before the uncertainty is resolved that surrounds the influence of human intervention in climate system due to emissions of greenhouse gases and aerosols. The main sources of emissions for most of these gases are associated with energy activities. This and other environmental concerns are yet another reason why the direction of technological changes in the energy systems is important. Some policies that lead to radically lower future emissions would need to be implemented before the uncertainty about possible climate change is resolved so as to reach sufficient cumulative experience with these technologies in time and so facilitate their future diffusion.

One of the important motivations for developing this new approach for endogenizing technological learning and uncertainty in energy systems scenarios was to capture the different directions of possible future technological change as a result of many technology replacements and incremental improvements. Our basic assumption is that endogenous learning is a function of cumulative experience measured by cumulative installed capacity and that this process is uncertain. Clearly, this is a strong oversimplification. There are many other indicators of technological learning but we chose this one because it is relatively easy to measure. Nevertheless, we feel that the oversimplification is warranted as a tool for analyzing the cumulative effect of incremental investments in new technologies on shaping alternative future directions of energy systems development.

Energy services are expected to increase dramatically especially in the now developing countries during the next century. This also means that the installed capacities of energy extraction, conversion, transport, distribution and end-use technologies are going to increase accordingly, perhaps at a somewhat lower rate due to the overall improvements of efficiencies throughout the energy system as older technologies are replaced by newer vintages. Here again, the alternative directions of energy systems development are important. They will determine to a large extent the eventual energy requirements that are needed to fulfill this increasing quest for energy services. The actual energy requirements for a given provision of energy services can range from very high to extremely low compared with current standards. In a similar way, 

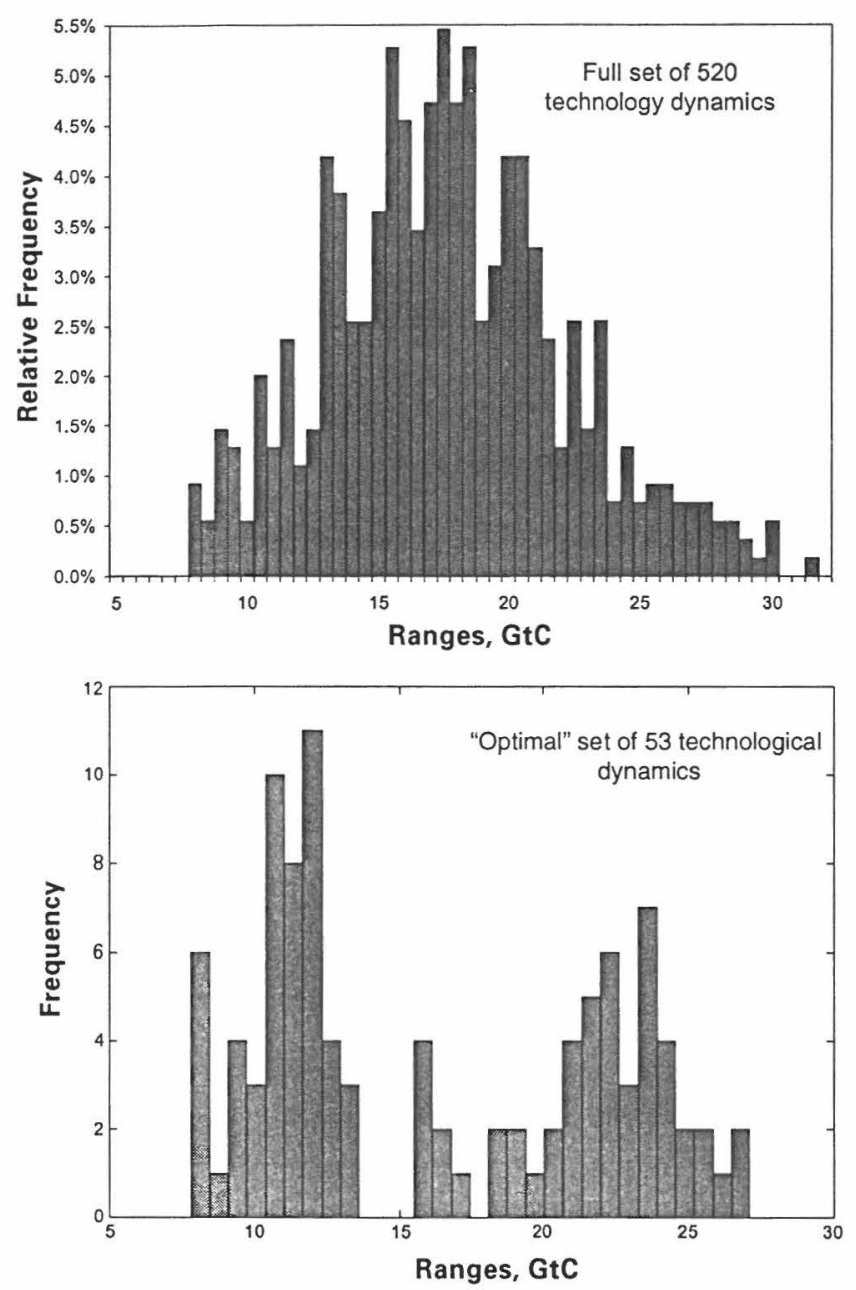

Fig. 1. Global carbon dioxide emissions range for the full set of 130,000 scenarios with endogenous technological change comprising some 520 different technology dynamics against the range of more than 13,000 "optimal" scenarios from 53 different technology dynamics. All scenarios share a given useful energy trajectory, emissions range in GtC.

the future environmental impacts of energy systems would vary accordingly as well. For example, carbon dioxide emissions decreases from 10 times the current levels to virtually no net emissions by 2100 for scenarios in the literature. Fig. 1 shows the range of future carbon dioxide emissions for the full set of 520 technological dynamics (some 130,000 scenarios) against the set of 53 "optimal" dynamics (more than 13,000 scenarios). In comparison Fig. 2 shows the range of emissions for some 400 scenarios from the published literature collected for the new IPCC Special Report on Emissions Scenarios (Morita and Lee, 1998; Nakićenović et al., 1998b). The emissions range from 7 to $41 \mathrm{GtC}$ by 2100 compared to about $6 \mathrm{GtC}$ in 1990 . These figures illustrate that the set of scenarios developed for capturing endogenous technological learning and uncertainty covers most of this range. The scenarios from the literature span this range due to the variation of the driving forces of future emis-

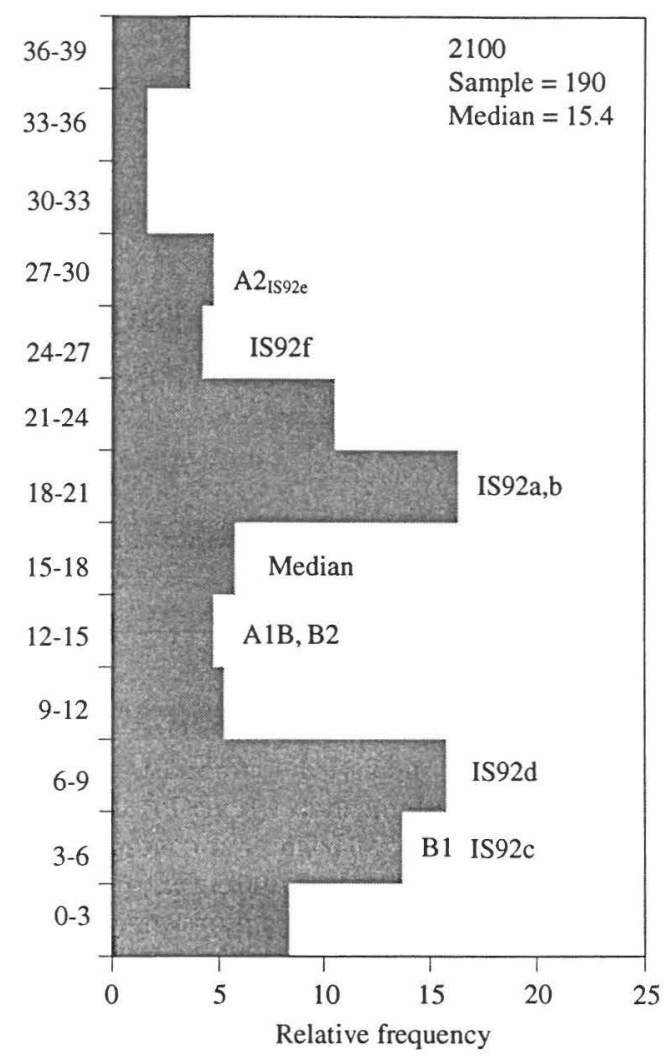

Fig. 2. Global carbon dioxide emissions for the range of some 400 scenarios from the literature, emissions range in GtC (Morita and Lee, 1998; Nakićenović et al., 1998b).

sions such as the energy demand. In contrast, the set of scenarios with endogenous learning spans the range due to different technological dynamics alone. It is interesting to note that the "optimal" scenarios match quite closely the distribution of the scenarios from the literature but with a somewhat narrower range (they leave the extreme tails of the distribution uncovered). In contrast, the frequency distribution of the full set of 520 technology dynamics is different from the other two with many more scenarios in the mid-range of the distribution. This means that the optimal or most "cost-effective" development paths correspond quite closely to the scenario distribution from the literature. The "median" or "central" futures are underrepresented both in the literature and among the scenarios indicating that there appears to be a kind of "crowding-out" effect surrounding balanced and median type of scenarios. In any case, technological learning as specified in our approach leads to future energy systems that are marked either by high or low emissions ranges with one single useful demand trajectory demonstrating a kind of implicit bifurcation across the range of possible emissions.

In order to simplify matters, we have assumed one single trajectory of global useful (end-use) energy requirements as an input assumption for all 130,000 scenarios considered in this analysis. What is varied endogenously 


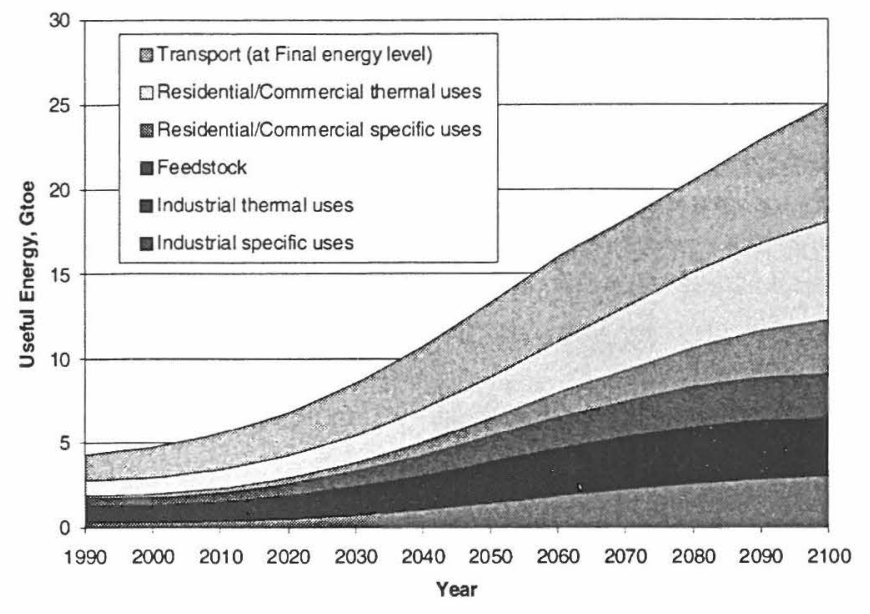

Fig. 3. Global useful energy demand trajectory common to all 520 different technology dynamics (130,000 scenarios), in Gtoe.

are technologies that comprise the energy system and their costs. Fig. 3 shows the single useful energy trajectory that is common to all scenarios. It represents relatively high useful energy demand compared with the scenarios in the literature. However, it is associated with the considerable variations of final and primary energy demand trajectories across the scenarios. It shows that a very wide portfolio of future energy systems characteristics is consistent with one single end-use demand trajectory. The scenarios map the higher part of the range of future primary energy requirements found in the scenario literature, but do leave the lower part of the range uncovered (that is associated with very low demand scenarios in the literature). As mentioned, they do cover most of the emissions range.

\section{Increasing returns and uncertainty}

Time horizons of a century or more are frequently adopted in energy studies. Modeling energy systems developments over such long-time horizons impose a number of methodological challenges. Over longer horizons technological change becomes fluid and fundamental changes in the energy system are possible. Especially difficult has been to devise an appropriate representation of endogenous technological change and the associated uncertainties. In general, induced technological change and uncertainties are interconnected. It is widely recognized that they jointly play a decisive role in shaping future energy systems. Many approaches to model these processes have included elements of increasing returns to scale and decreasing uncertainty to scale. This basically means that technologies improve with cumulative experience as expressed by the scale of their application. Costs and uncertainty are assumed to decline with increasing scale of application. Learning or experience curves are a characteristic representation of such processes.

In contrast, the "standard" modeling approaches with diminishing returns do not allow for such consequences of technological learning processes. Despite this deficiency, the diminishing returns dominate the standard economic theory. Perhaps, this is due to the very elegant and simple concept of equilibrium that can be achieved under those conditions. Diminishing returns to scale generate negative feedbacks, which tend to stabilize the system by offsetting major changes and produce inevitably a unique equilibrium independent of the initial state of the economy. In mathematical terms, the models are convex and lead generally to unique solutions.

Increasing returns on the other hand lead to disequilibrium tendencies by providing positive feedbacks. After (generally large) initial investments in RD\&D and early market introduction, the incremental costs of further applications become cheaper and cheaper per unit capacity (or as assumed here, per unit output). Thus, the more widely adopted a technology, the cheaper it becomes (with lower uncertainties, leading to lower risks to adoption). There are many incarnations of this basic principle. One of the more well-known ones is the concept of "lock-in". As technology becomes more widely adopted it tends to increasingly eliminate other possibilities. Thus, the lock-in. Another concept frequently used in empirical analysis is the so-called learning or experience curve. At the core of all of these processes is the technological learning - the more experience is gained with a particular technology, the larger are the improvements in performance, costs and other important technology characteristics.

Despite the fundamental importance of technological learning, the modeling of these processes has not received the necessary attention in the literature. Several reasons may explain apparent lack of systematic approaches. Among them, the complexity of appropriate modeling approaches is perhaps the most critical one. Increasing returns to scale lead to non-convexities so that the standard optimization techniques cannot be applied. In conjunction with the treatment of uncertainties, modeling of technological learning becomes methodologically and computationally very demanding. It requires the development of the so-called global non-smooth stochastic optimization techniques. They are only now under development (Ermoliev and Norkin, 1995, 1998; Horst and Pardalos, 1995).

Fig. 4 gives learning or experience curves for three technologies that generate electricity. Costs of unit-installed capacity are shown against cumulative-installed capacity. The lowest curve shows the improvement of gas turbines. Today, they are the most cost-effective technology for electricity generation. This was certainly not the case three decades ago. The costs were high and it was by no means certain that the great technology 


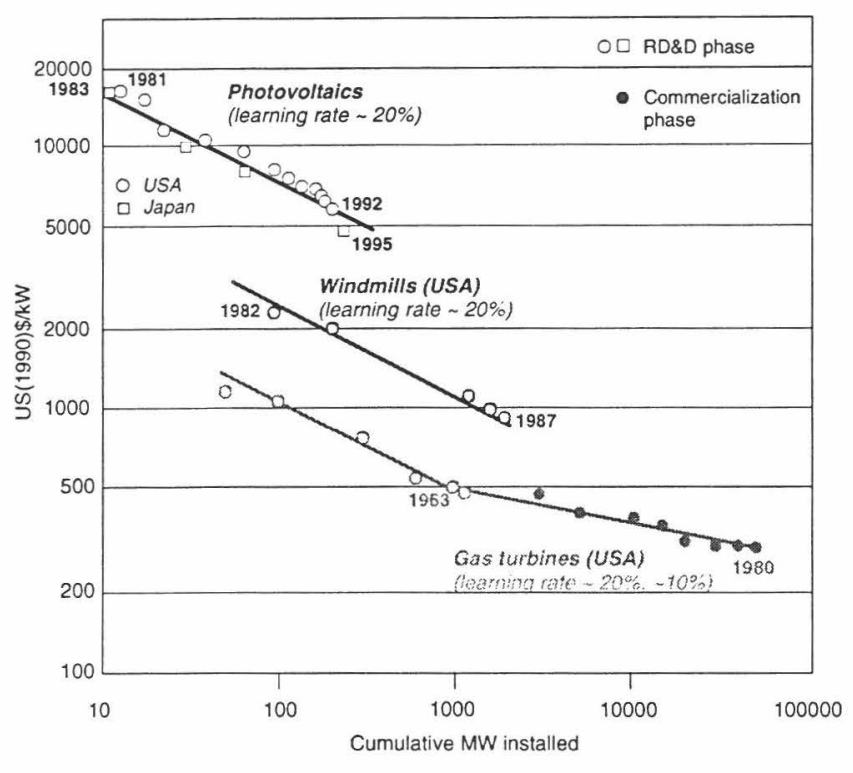

Fig. 4. Technology learning curves for three different electricity generation technologies - gas turbines, windmills and photovoltaics. Cost improvements per unit installed capacity, in US(1990)\$ per kWe, are shown against the cumulative installed capacity, in MWe, on logarithmic scale (Nakićenović et al., 1998a; MacGregor et al., 1991; Christiansson, 1995).

improvements would be achieved as that the curve suggests. Until early 1960s, the technology can be characterized as "pre-commercial". The costs were very high and the improvement rates were particularly rapid, about $20 \%$ reduction in unit costs per doubling of cumulative capacity. Thereafter, the improvement rate declined and has averaged to less than $10 \%$ per doubling. This development phase was no doubt also associated with significant reduction in uncertainties. In the early development phases, the investments in this technology were indeed risky as many accounts indicate.

Fig. 4 also shows two relatively new electricity generation technologies. Wind power is becoming "commercial" technology in many parts of the world especially where wind is abundant. Typical examples are wind application in Denmark. The cost reductions are impressive with about $20 \%$ per doubling of cumulative capacity. However, wind is on an average significantly costlier than gas turbines as source of electricity. Risk is also higher. In contrast, photovoltaics portray equally impressive performance improvements of about $20 \%$ unit costs reductions per doubling, but from a very high level of costs. They are about an order of magnitude more expensive than gas turbines per unit capacity. The future prospects are thus very promising but they are also associated with great risks for potential investors.

The learning curves were used in stylized form in a number of energy modeling approaches to capture elements of endogenous technological change. At IIASA, Messner (1995) incorporated learning curves for six electricity technologies in the simplified version of the

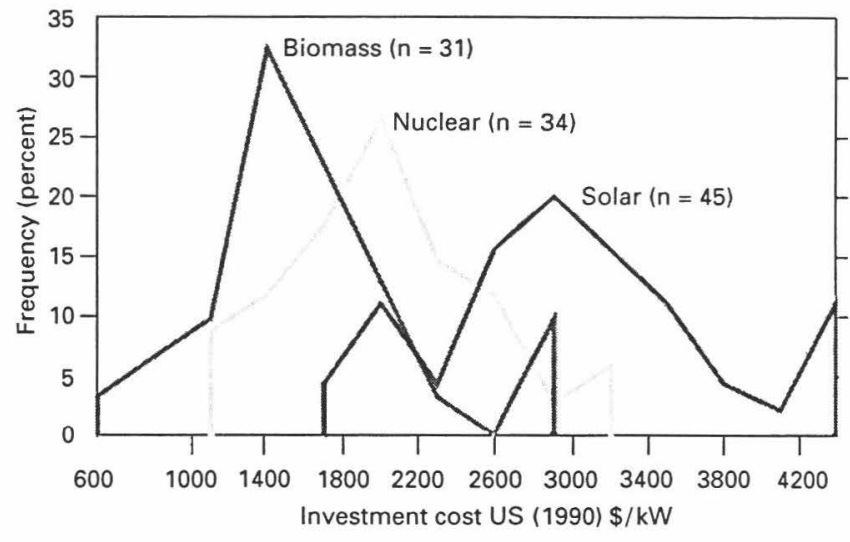

Fig. 5. Range of future investment costs distributions from the IIASA technology inventory for biomass, nuclear, and solar electricity generation technologies, in US(1990)\$ per kW (Messner and Strubegger, 1991; Nakićenović et al., 1998a).

(deterministic) energy systems-engineering model MESSAGE. This is a linear programming framework, so that integer programming was needed to deal with emerging non-convexities in the problem formulation. It was assumed that the "new" energy technologies have a certain cost reduction per each doubling of cumulative installed capacity ${ }^{1}$ The approach was very innovative and has led to a number of important insights for further modeling of endogenous technological change (Grübler and Messner, 1996; Nakićenovic, 1996, 1997). However, the major drawback was the significantly higher complexity and very high computational demands. Another important deficiency of the approach was that the learning rates were deterministic. MESSAGE is a model with perfect foresight, so that early investments in new, costly technologies were always rewarded with increasing returns. Yet, it is clear that such reductions are possible on average but with a considerable degree of uncertainty.

The next step at IIASA was to introduce uncertainties in the distributions of future costs. The basis for this approach was the IIASA technology inventory that now contains information on some 1600 energy technologies, on their costs, technical and environmental characteristics (Messner and Strubegger, 1991). Fig. 5 gives an example of future cost distributions of three energy technologies from the inventory (Nakićenovic et al., 1998a). It illustrates that the distributions are not symmetric and that they have very pronounced tails with both very "pessimistic" and "optimistic" views on future costs per

\footnotetext{
${ }^{1}$ Cost reduction or the so-called learning rate may be quite different depending on how "learning" is measured. As mentioned, the learning rate for photovoltaics in Fig. 4 is about $20 \%$ per doubling of cumulative capacity. For example, Watanabe (1995) analyzed direct investment in photovoltaics in Japan indicating that the unit costs decreased by about $50 \%$ per doubling of cumulative investment. Grübler (1998) estimates the learning rate at $30 \%$ per doubling of cumulative installed capacity based on the same data set from Watanabe (1995).
} 
unit capacity. Such cost distributions were introduced explicitly in a simple, stochastic version of MESSAGE and have lead to spontaneous "hedging" against this uncertainty as an emerging property of the model (Golodnikov et al., 1995; Messner et al., 1996). Finally, both approaches of endogenous learning and uncertainty were combined for a very highly stylized stochastic version of MESSAGE with increasing returns for "just" three "technologies" (Grübler and Gritsevskyi, 2000). One was characterized with no learning what so ever. Another technology displayed moderate learning of about $10 \%$ per doubling and the third with much more rapid $20 \%$ per doubling. The latter two learning rates were associated with uncertainties that were based on the above future-cost-distribution functions. In this much more complicated approach, the diffusion of new technologies occurred spontaneously and displayed S-shaped patterns so characteristic for technological diffusion. This occurred without any other explicit technology inducement mechanisms other than uncertain learning and hedging. The disadvantage of the approach was that it was very computationally demanding and basically infeasible for application with many technologies as is required for development of long-term energy scenarios.

Here we retain this basic approach and combine technological leaning with uncertain outcomes while significantly extending the application to a hundred technologies. This is possible due to the application of new global non-smooth stochastic optimization techniques in conjunction with "parallel" problem structure and computing techniques. Cost reductions are assumed to be uncertain and are thus not specified by a given deterministic learning rate value. The learning rates are uncertain and are captured by assumed distribution functions. We assume that the generic cost reduction function has the following form:

$$
C I_{t}=\left(2^{-\beta}\right)^{N D_{t}},
$$

where $C I_{t}$ is the cost reduction index, or the ratio between technology unit costs (or more precisely, the annual levelized costs) at time $t$ and initial cost in the base year; $N D_{t}$ is the number of doublings of cumulative output achieved by time $t$ compared to the initial output; and $\beta$ is the progress ratio that indicates the cost reduction rate per doubling of the output. $\beta$ is a random variable with a known distribution function. We have assumed that $\beta$ is normally distributed with known mean and variance. It is important to note that the suggested algorithmic approach is not limited to the type of distribution assumed here, and, in fact, that it does not require any prior knowledge about type of the distribution function. ${ }^{2}$

\footnotetext{
${ }^{2}$ We need to have ability to compute mean value for corresponding distribution and to produce random samples based on that distribution. Implementation in the form of "black box" is perfectly suitable.
}

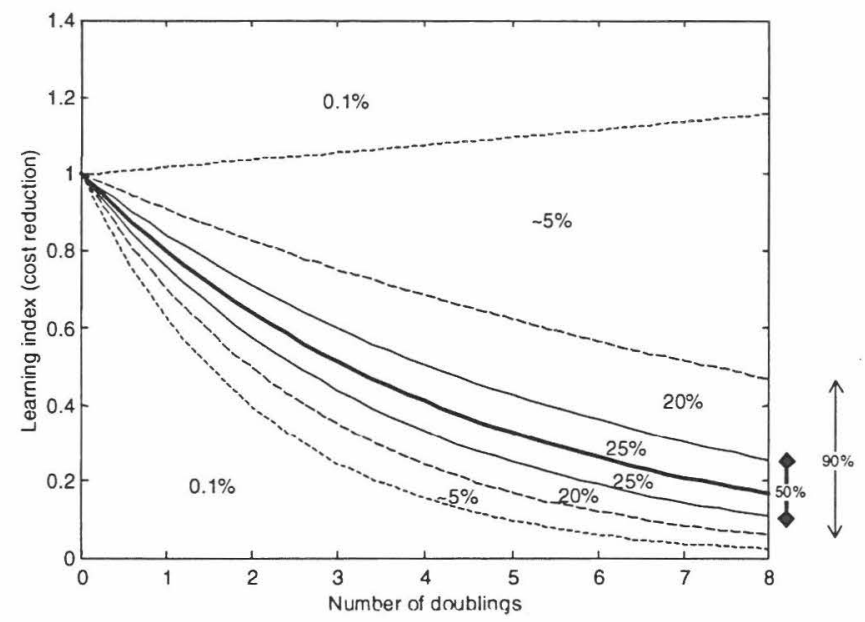

Fig. 6. Uncertain cost reductions represented by the learning index as a function of number of doublings (of cumulative output). The expected value of $\beta$ (the mean) learning index (rate), corresponds to $20 \%$ cost reduction per each doubling (of cumulative output). The numbers between the isolines of different learning indices indicate probability ranges. There is a small probability of no learning at all between any given doubling.

Fig. 6 illustrates the uncertain learning index as a function of each doubling (of cumulative output). The expected value for the cost reductions rate is $20 \%$ per doubling in the example shown. The numbers between the isolines indicate the probability ranges of occurrence of different learning rates. For example, there is a $50 \%$ chance that the cost reductions rate falls between 14 and $25 \%$ per each doubling. Please note that there is a small chance of $5 \%$ that the cost reductions would range from very small to actual cost increase and that there is a very small probability of $0.1 \%$ that there would be significant cost increase per each doubling. This indicates a real possibility of negative learning or "induced forgetting" rather than learning. Such representation of uncertain learning illustrates the true risk of investing in new technologies. There is a high chance that technology would improve with accumulated experience, but there is also a small chance that it would be a failure and even a smaller chance of a genuine disaster.

We extend here the application of uncertain learning to many new technologies ranging from wind and photovoltaic to fuel cells and nuclear energy. In keeping with the earlier approaches to capture learning at IIASA, we assume that traditional, "mature" technologies do not benefit from learning (another interpretation is that cost reductions as the result of learning are insignificant compared to other uncertainties that affect costs). Altogether there are 10 clusters of new technologies that benefit from induced learning.

As already mentioned, we assume in addition that all technologies, traditional and new ones, have stochastic costs with known distributions in any given period 
(similar to the distributions of electricity generation technologies used in Golodnikov et al., 1995). The difference is that we assume that cost distributions of traditional technologies are static over time and the costs in different time periods are independent random values. For new technologies, due to possible cost reductions to learning (as described above), the costs are defined by conditional probabilities that result from the realization of a particular value for the uncertain learning rate. Again just for reasons of simplicity, we assume that all initial cost distribution are log-normal with different mean and variance based on the empirical analysis of technological characteristics with the IIASA technology inventory (see Strubegger and Reitgruber, 1995).

We assume that the cost distribution function for each of the new technologies at any given moment of time $t$, under the condition that $N$ doublings of cumulative output have been achieved and that the realized value for random learning rate $\beta$ is equal to $b$, is defined by the following expression:

$$
\begin{gathered}
F_{t}\left(\zeta \mid N D_{t}=N, \beta=b\right)=F_{0}\left(m_{t}, s_{t}\right), \\
m_{t}=m_{0}\left(2^{-b}\right)^{N}, s_{t}=K m_{t},
\end{gathered}
$$

where $F_{0}(\cdot, \cdot)$ is the initial log-normal distribution function with parameters $m_{0}$ and $s_{0} ; K$, is the ratio between the standard deviation and the expected mean value and defines the compactness of the distribution. It is assumed to be a function of typical unit size (for bigger unit size $K$ is bigger). We decided to keep $K$ constant over time due to the lack of empirical data, so it can be obtained simply by solving the following equation:

$$
s_{0}=K m_{0},
$$

where $m_{0}$ and $s_{0}$ are derived empirically from statistical analysis. $^{3}$

A new feature of our approach (in addition to the uncertain learning rates) is that the future costs of all technologies are uncertain and assumed to be distributed according to the log-normal distribution. These are stylized distribution functions that, as was mentioned, reflect indirectly the costs distributions of energy technologies in the future based on the analysis of the IIASA energy technology inventory. In addition, the mean value of these cost distributions is assumed to decrease and variance to narrow with increasing application of new technologies according to the generic cost reduction

\footnotetext{
${ }^{3}$ Suggested technique does not require or utilize specific relation between $F_{t}$ and initial distribution $F_{0}$. It also does not need to keep $K$ constant over time. In absence of better understanding of quite complex and non-linear relationship and due to the luck of empirical data we decided to use the most simple assumptions one can make - the type of distribution stays the same (distribution does not change its shape), mean value follows realized cost reduction curve, variance goes down proportionally to expected cost reduction.
}

function (specified above) with normally distributed progress ratio. This means that the process of technological learning is uncertain even as cumulative experience increases. The uncertainty of new technologies is characterized with the joint distribution of cost uncertainty and learning uncertainty. In summary, we assume both uncertain future costs for all technologies and uncertain learning for new technologies.

Another uncertainty considered here is associated with magnitudes and costs of energy reserves, resources and renewable potential and their extraction and production costs. Following the estimates by Rogner (1997), Nakićenović et al. (1996) and others, we assume a very large global fossil resource base corresponding to some 5000 Gtoe and accordingly large renewable potentials. We also assume that the energy extraction and productions costs are uncertain varying by a factor of more than five. Following the approach proposed by Rogner (1997) we formulated aggregate, global, upward-sloping supply curves with uncertain costs. Thus, the supply of fossil and non-fossil energy sources is characterized by expected increasing marginal costs and is one of the few areas where we have not assumed increasing returns, but we did assume uncertain costs.

\section{Technological spillovers}

Technologies are related to each other. For example, jet engines and gas turbines for electricity generation are related technologies. In fact, the latter were initially derived from the former. These kinds of relationships among technologies are frequent. They imply that improvement in some of the technologies can be transferred to other related technologies. For example, improvements in automotive diesel engines might lead to better diesel-electric generators because the technologies are closely related to each other. The improvements in one area that lead to benefits in other areas are often referred to as spillover effects. In case of related technologies this is a real possibility. For example, we consider the different applications of fuel cells such as for stationary electricity generation and for vehicle propulsion. We also consider fuel cells that have the same end-use application but different fuels, e.g. hydrogen and methanol mobile fuel cells. These fuel cells are different but they are related in the technological sense so that improvements in one technology may lead to improvements in the other. In this new approach to model technological learning and uncertainty, we explicitly consider the possibility of such spillover effects among energy technologies.

However, operational implementation of spillovers is not trivial. One of the important barriers is the lack of technology "taxonomy". Presumably, the possibility of positive spillovers from technological learning is higher for technologies that are similar compared to those that 
Table 1

Ten technology "clusters" that group new energy technologies that are assumed to benefit from spillovers associated with technological learning induced from one technology to another within the cluster

\begin{tabular}{|c|c|c|c|c|}
\hline Name & $\begin{array}{l}\text { Expected } \\
\text { learning } \\
\text { rate }(\%)\end{array}$ & $\begin{array}{l}\text { Cumulative } \\
\text { output at } \\
\text { base year } \\
\left(G W y r^{a}\right)\end{array}$ & $\begin{array}{l}\text { Typical } \\
\text { lifetime } \\
\text { (yr) }\end{array}$ & Technologies \\
\hline Fuel cells in transportation ${ }^{b}$ & 20 & 0.01 & 10 & $\begin{array}{l}\text { Hydrogen-, liquid-hydrogen- and methanol- } \\
\text { based fuel cells (FC) in transportation, three tech- } \\
\text { nologies }\end{array}$ \\
\hline $\begin{array}{l}\text { Decentralized fuel cells (Industrial and } \\
\text { residential \& commercial sectors) }\end{array}$ & 20 & 0.1 & 20 & $\begin{array}{l}\text { Hydrogen-based FC in Industrial and } \mathrm{R} \& \mathrm{C} \text { sec- } \\
\text { tors, two technologies }\end{array}$ \\
\hline Centralized fuel cells in energy sector ${ }^{d}$ & 20 & 1 & 30 & $\begin{array}{l}\text { Natural gas- and coal-based large-scale FC, two } \\
\text { technologies }\end{array}$ \\
\hline Solar photovoltaic & 25 & 0.2 & 20 & $\begin{array}{l}\text { Solar panels in industrial and } \mathrm{R} \& \mathrm{C} \text { sectors and in } \\
\text { energy sectors, three technologies }\end{array}$ \\
\hline Solar to hydrogen & 10 & 1 & 20 & Solar to hydrogen production, one technology \\
\hline Nuclear high-temperature & 10 & 10 & 40 & $\begin{array}{l}\text { Nuclear high-temperature reactors with hydrogen } \\
\text { output, one technology }\end{array}$ \\
\hline Wind & 15 & 2 & 30 & Wind power generators, one technology \\
\hline Synthetic fuels and hydrogen production & 20 & 3 & 30 & $\begin{array}{l}\text { Synthetic fuels and hydrogen production from } \\
\text { biomass, gas and coal, six technologies }\end{array}$ \\
\hline Liquid hydrogen production & 10 & 0.5 & 30 & Hydrogen liquefaction, one technology \\
\hline
\end{tabular}

a Part of model assumptions. In many cases, there is no reliable statistics for global cumulative output.

${ }^{b}$ Contribute to other fuel cells clusters with weight and accelerated by input from stationary units with weights 0.1 and 0.01 for decentralized and centralized installation correspondingly.

${ }^{\mathrm{C}}$ Contribute to other fuel cells clusters with weight 0.5 to centralized units and 0.1 to transportation and accelerated by input from centralized units with weight 0.1 and with 0.5 for transportation.

${ }^{d}$ Contribute to other fuel cells clusters with weight 0.1 to decentralized units and 0.01 to transportation and accelerated by input from decentralized and transportation units with weight 0.5 .

are not. Thus, some kind of measure or metric of technological "proximity" or "distance" is required even though a genuine taxonomy does not exist. A number of proposals have been made that could conceivably lead to the development of a taxonomy in the future (Foray and Grübler, 1990). Instead of venturing here in more complex representations of technology relationships, we simply assume that there are basically two explicit types of spillover effects. One is indirect through the connections among energy technologies within the energy system. For example, cheaper gas turbines mean cheaper electricity so that ceteris paribus this could favor electricity end-use technologies for providing a particular energy service compared to other alternatives. The other effect is more direct. Some technologies are related through their "proximity" from technological point of view as was suggested by the example of hydrogen and methanol mobile fuel cells. We explicitly define "clusters" of technologies, which may lead to spillovers from learning in one technology to another. Within clusters, the spill- over effects are assumed to be strong and weak across clusters.

Technology clusters were explicitly pre-specified. Table 1 shows the groupings of technologies into 10 clusters. Each cluster consists of technologies that are related either because they are technologically "close" (i.e., are similar) or because they enable each other through the connections among them within the energy system.

The nature of the spillover effects within and across clusters is assumed to be different. Technologies from the same cluster share total cumulative output and are assumed to have the same learning rate, but their actual costs are drawn independently from their respective distributions.

Fig. 7 illustrates the spillover effects within one cluster of technologies. The shown example gives two density functions of technology costs in 2030 for centralized fuel cells. The density function with lower overall costs is for the case of spillover effects within the technology cluster 


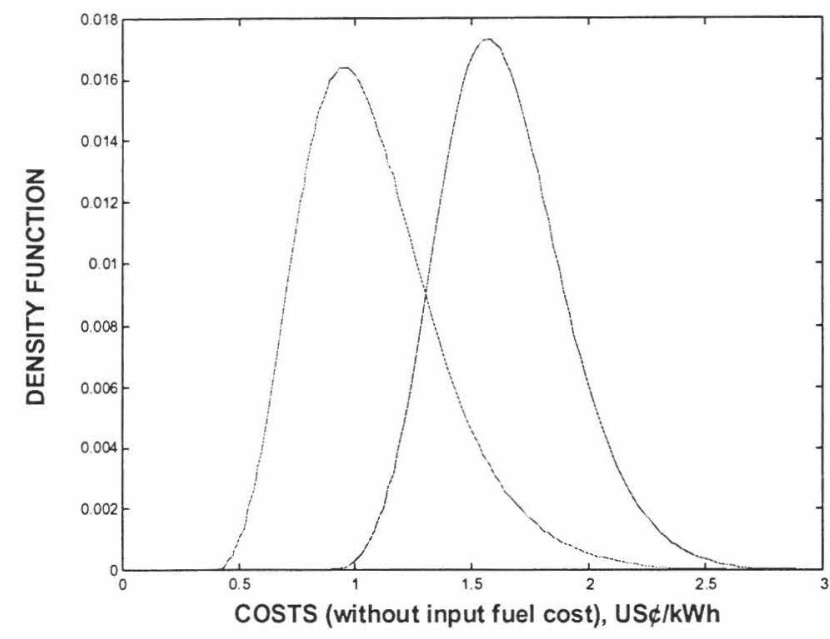

Fig. 7. Spillover effects within the cluster of decentralized fuel cell technologies. Two density functions of fuel cell costs in 2030 are shown, in US(1990)d per kw h without fuel costs. The density function with lower overall costs is for the case of spillover effects within the technology cluster and the one with higher overall costs is for the case without spillover effects.

and the one with higher overall costs is for the case without spillover effects. The costs are given in US (1990)\& per kW h of electricity generation without the fuel costs. Both the expected costs and their variance are substantially higher without the spillover effects. Thus, the costs are expected to be lower with spillover effects and as well as the uncertainty. The probability of lower costs is thus overall much higher with the spillovers.
However, the high tail of the density distribution is proportionally more pronounced in the case of the spillover. This is an interesting feature of these density functions. The expected costs are generally lower with spillovers, but the possibility of realizations of very high costs compared with the mean are higher at the same time. Thus, spillovers also amplify somewhat the small chance of induced "forgetting."

Spillover rates between clusters are proportional (weighted) to the technological "proximity", e.g. how close the technologies are related to each other. Examples include additive learning from all kinds of fuel cells, e.g. stationary and mobile. Another factor is that stationary fuel cells can contribute significantly into the learning for the mobile ones due to large capacity (size), and wise versa, that experimenting with small-scale mobile units could be an important factor that helps early development of stationary units.

Fig. 8 gives a schematic diagram of the 10 technology clusters and indicates how they are related to each other with respect to the assumed learning spillover effects within the structure of the energy system. Two of the technology clusters (also shown in Table 1) are characterized by generally large "unit size" compared to other technologies - nuclear high-temperature reactors (HTRs) and infrastructure clusters. Consequently, very large cumulative output is required for achieving a doubling compared to other clusters. This leads to correspondingly high risks in induced learning. The expected learning rates are indicated for each cluster. The modular (smaller "unit size") technologies have generally
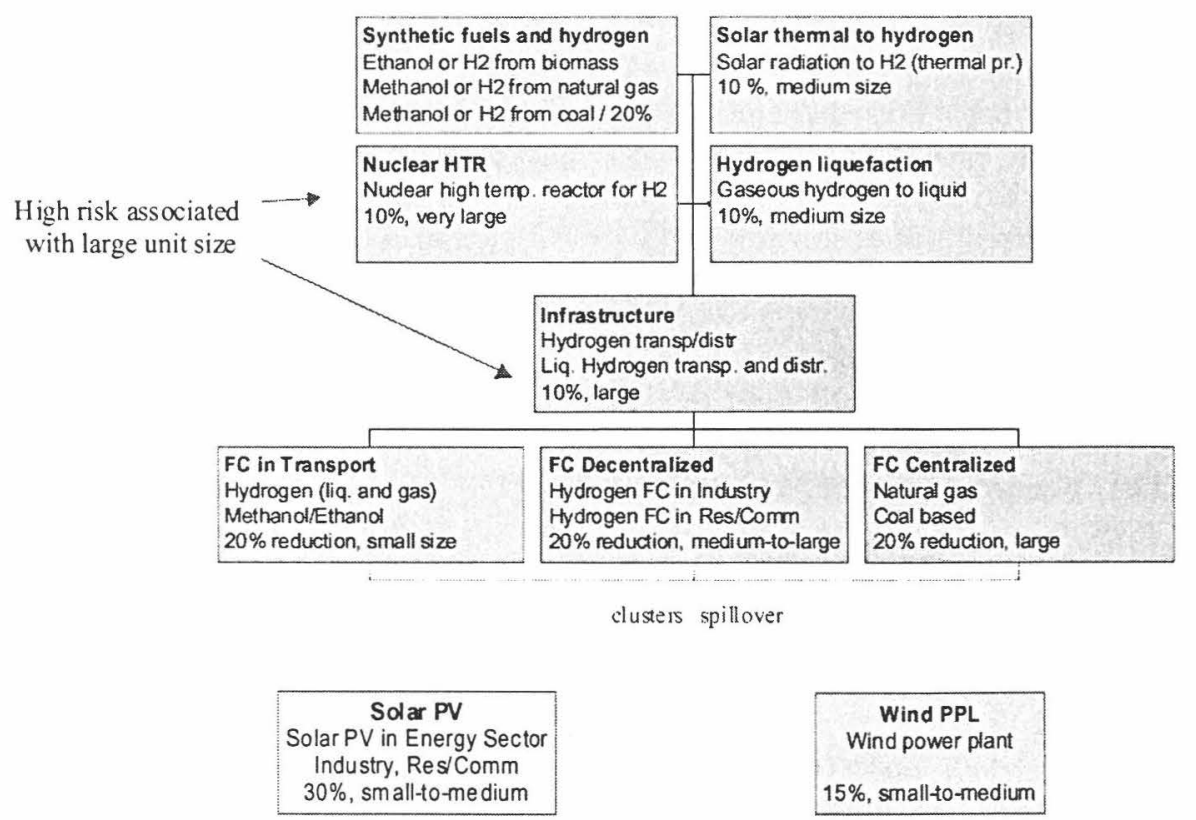

Fig. 8. Schematic diagram of the 10 technology clusters and their relationships to each other with respect to the assumed learning spillover effects within the structure of the energy system. Technologies of each cluster are listed together with their assumed expected mean learning rates. 
higher mean learning compared to other technologies. The highest mean learning rate is indicated for the photovoltaics cluster, the lowest are shared by the solarthermal (hydrogen), nuclear (HTR) and infrastructure clusters.

\section{Model structure and implementation}

Any realistic policy in the presence of uncertainties bears risks, in particular, risk of underestimating or overestimating future technology costs. Explicit introduction of these risks creates a driving force for the development of new technologies necessary for making the energy system flexible enough against possible instabilities and surprises. Thus, uncertainty of future technology costs and characteristics in itself induces technological change. When this uncertainty is broadened to include technological learning and spillovers, the complex interplay between all of these three mechanisms leads to patterns of technological change that are encountered in deterministic modeling approaches as well but under the conditions of exogenous constraints. The difference is that here this behavior is the result of induced technological change that occurs "spontaneously" due to stochastic nature of technological learning within the energy system.

The conventional approaches of the control theory are applicable only in the case of small number of variables (e.g. for simple energy systems), since they deal with unrealistically detailed long-term strategies attempting to provide the best choice for every combination of uncertainties and designs which may occur before the given time moment. This "chess game"-type concept of solution is essential for application of standard dynamic programming equations.

The same type of solution concept is used in multistage stochastic optimization models. Although the large-scale optimization techniques are used in such case instead of the recurrent equations, the actual size of solvable problems is small again. It is essentially connected with the concept of solutions, which requires the expansion of original finite-dimensional model to the model with infinite number of variables. Both approaches seem to be meaningful only for "on-line", or short-term energy planning problems. They are unrealistic for the analysis of long-term energy policies.

Since it is impossible to explore all details of long-term energy developments, our approach is based on the socalled two-stage dynamic stochastic optimization model with a rolling horizon. The concept of solution in this case depicts the ex ante path of developments, which is flexible enough for adjustments to possible ex post revealed uncertainties ("surprises"). The concept of rolling horizon requires adjustments of ex ante strategies each time when essential new information is revealed. A par- ticular type of this model was proposed by Ermoliev (1995) for the analysis of global change issues and is ideally suited for energy system-engineering analyses as represented in some applications of the MESSAGE model. Stochastic version of the MESSAGE (see Golodnikov et al., 1995) is also a two-stage dynamic stochastic optimization model. This model explicitly incorporates risks of underestimating costs, which leads to a convex, in general non-smooth, stochastic optimization problem.

Overall approach is based on the idea of representing energy systems development as a dynamic network where flows from one energy form to another correspond to energy technologies such as electricity generation from coal or gas power plants. Fig. 9 illustrates the assumed reference energy system as composed of about 100 different technologies. Five different stages of energy flows are shown - energy extraction from energy resources, primary energy conversion into secondary energy forms, transport and distribution of energy to the point of end use that results in the delivery of final energy, and finally the conversion at the point of end use into useful energy forms that fulfill the specified demands (as discussed above). All possible connections between the individual energy technologies are also specified in Fig. 9. Various demands for useful energy are shown for different sectors of the economy. Each technology in the system is characterized by levelized costs, unit size, efficiency, lifetime, emissions, etc. In addition to various balance constraints, there are limitations imposed by the resource availability as a function of (uncertain) costs. The overall objective is to fulfill various demands by the utilization of technologies and resources with the minimal total discounted system costs. ${ }^{4}$

In the case of known future costs, demands and other parameter values, it is possible to find a unique "optimal" solution for the evolution of the reference system shown in Fig. 9. It is obtained by solving the following deterministic, linear optimization problem

$$
\begin{aligned}
& \min \sum_{t=0}^{T} \mathrm{~d} s^{t}\left\langle C^{t}, x^{t}\right\rangle, \\
& B_{t} x^{t} \geqslant d^{t}, \quad t=0,1, \ldots, T, \\
& \sum_{k=0}^{t} R_{k} x^{k}=r, \quad t=0,1, \ldots, T, \\
& \sum_{k=0}^{T} P_{k} x^{k} \leqslant \mathrm{e}^{t}, \quad t=0,1, \ldots, T, \\
& 0 \leqslant x^{t} \leqslant \bar{x}^{t}, \quad t=0,1, \ldots, T,
\end{aligned}
$$

where $x^{t}=\left(x_{1}^{t}, \ldots, x_{n}^{t}\right)$ are activity levels of technologies and resources at time $t ; B_{t}$ is matrix of input and output relations among the technologies and $d^{t}$ is the demand

\footnotetext{
${ }^{4}$ Similar to many other models, a $5 \%$ discount rate was adopted.
} 


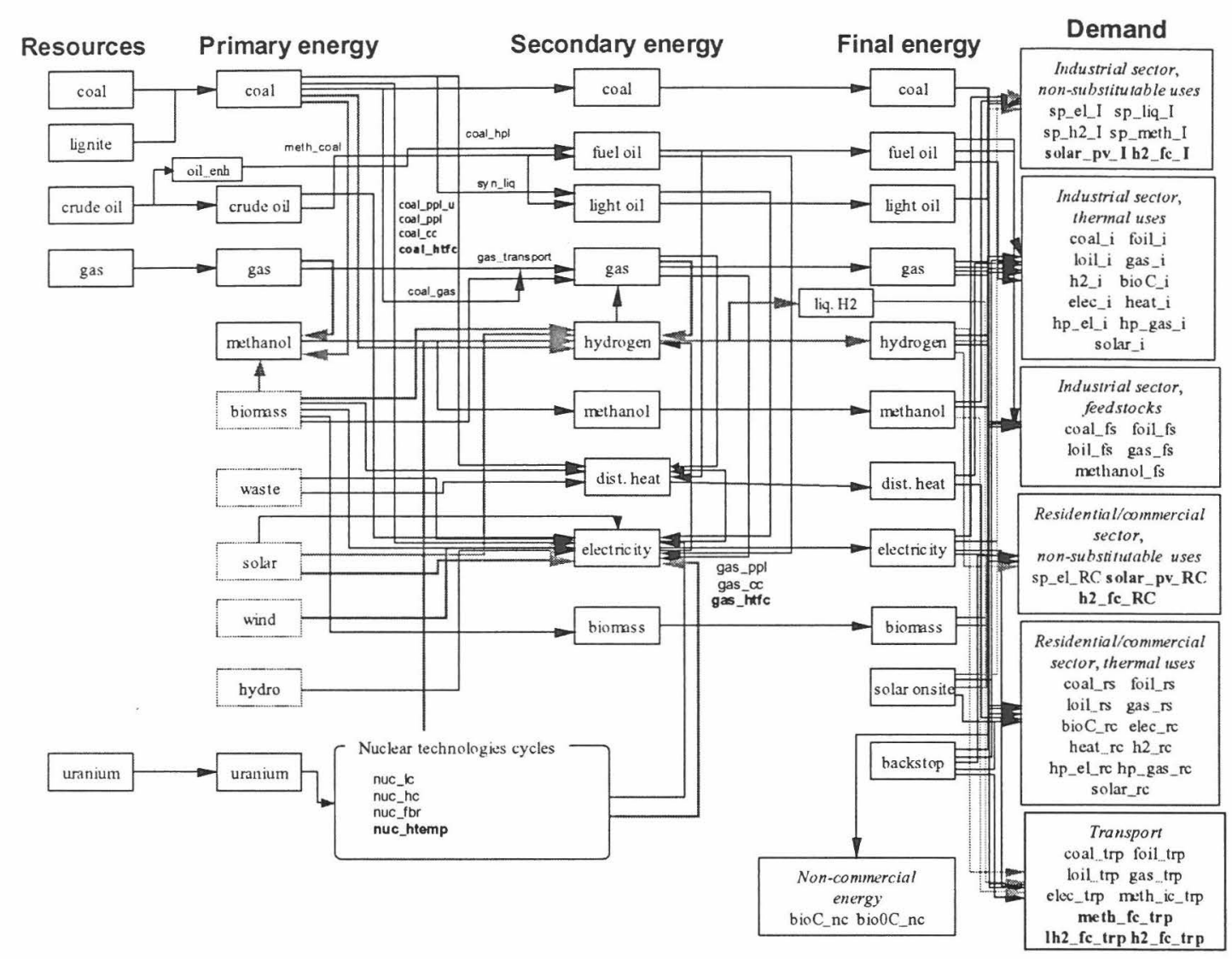

Fig. 9. Schematic diagram of the reference energy system showing some hundred individual technologies.

vector; $R_{t}$ is matrix for approximating the quadratic costs of resources and balances for resource use, $r$ are corresponding quantities; $P_{k}$ is the matrix of systems constraints, like market penetration constraints and maximum shares of specific resource and technology activities, $\mathrm{e}^{t}$ are corresponding limits; and $\bar{x}^{t}$ are the upper limits on technological activities.

Such deterministic formulations of future energy systems development result in highly restrained possibilities. In addition, the dynamics of future developments are prescribed by the system of assumed constraints. In contrast, there is a wide possibility of alternative future developments of the energy systems especially in the long run, over the scale of a century. This is amply demonstrated in the enormous range of future energy requirements and carbon dioxide emissions across the range of energy scenarios in the literature (see Fig. 1 above).

In contrast, the alternative formulation of the problem proposed here is highly unrestrained and "open." We assume that there is a priori "freedom of choice" among fundamentally different future structures of the energy system and possible future dynamics. The uncertainty is resolved through a simultaneous drawing from all distributions from each particular technology dynamics (see the Boxes 1 and 2 on Terminology). In order to make a rational choice among alternative technology dynamics, they are compared on a basis of expected system costs and risks associated with each particular technology dynamics. Risks or benefits are defined here as functions of the difference between the expected and realized costs of each technology dynamics. There are a number of alternative ways to do a risk quantification (see, for example, Markowits, 1959). We adopt a technique whereby the risk is represented by piece-wise linear functions of the following form:

$$
\begin{aligned}
& \min E \sum_{t=0}^{T} \mathrm{~d} s^{t}\left\langle C^{t}(\omega), x^{t}\right\rangle+E R\left(C^{t}(\omega),\left.x\right|_{0} ^{t}\right) \\
& =\sum_{t=0}^{T} \mathrm{~d} s^{t}\left\langle E C^{t}(\omega), x^{t}\right\rangle \\
& \quad+r_{i} \sum_{i=0}^{l} E \max \left\{0,\left\langle C^{t}(\omega)-E C^{t}(\omega)-\Delta \hat{c}_{i},\left.x\right|_{0} ^{t}\right) .\right.
\end{aligned}
$$

This, clearly asymmetric, form of the risk function has an obvious advantage over a more standard approach that is based on variance minimization. Splitting risk function into to two parts, that represent risk associated with cost 
underestimation. ${ }^{5}$ and the benefit associated with cost overestimation, i.e., a situation where costs turn out to be lower than expected, is a natural reflection of highly asymmetric risk perception of "loses" and "gains". Moreover, different "actors" (energy agents) may have quite different risk aversion. This approach allows, in principle, for the representation of different risks perceived by different decision actors or agents.

This asymmetric treatment of "risk" and "benefit" significantly increases the complexity of the problem - risk cannot be expressed just in terms of a functional relation of expected values and corresponding variances, like it is done in the case of a Markowitz's formulation. Formally speaking, the objective function specified above is nonsmooth and, obviously, highly complicated non-convex function defined on probabilistic space. In general form, it is analytically intractable problem even in case of relatively small system when just few technologies are considered. The problem could be solved by involving stochastic approximation technique (see Ermoliev and Norkin, 1995). This stochastic approximation approach is based on the idea of estimating solution of the original problem by solving another stochastic problem where original probabilistic space is replaced by finite (sufficiently large) number of simultaneously generated "samples" according to the distribution function for uncertain parameters (see Grübler and Gritsevskyi, 2000). This approach is significantly different from conventional Monte-Carlo approach. All drawings are performed simultaneously and resulting policy conclusion is formulated against all of considered outcomes. There are strong methodological similarities between the so-called exploratory modeling (see Bankes, 1993; Lempert et al., 1996; Robalino and Lempert, 2000) and approach used here, although we are using a very different implementation and analysis technique.

A systematic approach for the aggregation of scenario-specific solutions into a robust solution is examined in Ermoliev and Wets (1988). These techniques require explicit characterization of scenario-specific solutions, which may lead to extremely large-scale optimization problems. Different stochastic optimization techniques deal with the design of robust solutions from a set of beforehand or sequentially simulated scenarios. In the latter case the stochastic optimization procedure can be

\footnotetext{
${ }^{5}$ There are more then one factor that could lead to underestimation of realized cost. For all new technologies (especially at early stage of development), even in case where cost reduction rate is equal or even better then expected, there is a significant cost fluctuation due to uncertainty associated with such cost distributions (high variance, heavy tails, and so on). High dependence on particular resource form, low level of technological diversification and strong linkage between system parts, all these factors largely contribute to increasing probability of substantial cost underestimating. Such analysis would be close to impossibe to perform on a basis of simple cost-to-cost analysis for alternative energy supply chains.
}

\section{Box 1 on Terminology}

Scenario is a particular deterministic realization of a future energy system. Here it specifies unique values for all activity levels such as energy flows, increases in capacities, total systems costs, energy extraction, etc. Technological dynamics denotes here a more generic characterization of future developments with inherent uncertainties surrounding, for instance, future costs. A resolution of these uncertainties inherent in technological dynamics results in a given scenario. There is an infinite number of possible scenarios that share exactly the same technology dynamics. Thus, technology dynamics specifies a set of uncertain, generic relations. In particular, technological dynamics specifies here the set of uncertain costs reductions as a function of doublings of output, the cost distributions in any given period and possible spillover effects within and across the ten technology clusters within the reference energy system. Our approach to analyze and compare alternative technological dynamics is to assume specific distribution functions for uncertain parameters and relations. The uncertainty is resolved by a simultaneous drawing from all distribution functions for a given technology dynamics that then results in a deterministic scenario. After many such drawings, expected costs and other characteristics of the scenario sample for a particular technology dynamics and be estimated. The expected costs and other sample statistics and be used then for obtaining risk estimates associated with each technology dynamics. Each scenarios within the set belonging to one specific technology dynamics can be characterized by relative (conditional) probability relatively to others scenarios from that set. ${ }^{6}$ Feasible technology dynamics are those that satisfy given energy demands and other systems constrains. A run of scenarios refers to all scenarios generated from a given set of technology dynamics through simultaneous drawings from all uncertain distributions. In this application we analyzed 520 alternative technology dynamics and have drawn some 250 scenarios for each of them, resulting in a run of about 130,000 scenarios.

We call a given technology dynamics optimal (suboptimal) for given run if it is optimal (sub-optimal) in comparison to all other technology dynamics in the run of scenarios with respect to the weighted sum of its expected systems costs, and risk functions based on these costs, for all drawn scenarios.

Or in more formal way, the problem is given in box 2 .

\footnotetext{
${ }^{6}$ As we mentioned before each scenario has exactly zero probability of realization. It makes sense to talk about scenario probability under some conditions. For example, under conditions that from the set of $\mathrm{N}$ scenarios ONE should happened, it is possible to introduce and compare relative probability defined on this set of $\mathrm{N}$ scenarios.
} 
Box 2.

$$
\begin{aligned}
& \min E \sum_{t=0}^{T} \mathrm{~d} s^{t}\left\langle C^{t}\left(\left.x\right|_{0} ^{t}, \omega\right), x^{t}\right\rangle \\
& \quad+E R\left(C^{t}\left(\left.x\right|_{0} ^{t}, \omega\right), x^{t}\right)=\sum_{t=0}^{T} \mathrm{~d} s^{t}\left\langle E C^{t}\left(\left.x\right|_{0} ^{t}, \omega\right), x^{t}\right\rangle \\
& \quad+r_{i} \sum_{i=0}^{l} E \max \left\{0,\left\langle C^{t}\left(\left.x\right|_{0} ^{t}, \omega\right)\right.\right. \\
& \left.\quad-E C^{t}\left(\left.x\right|_{0} ^{t}, \omega\right)-\Delta \hat{c}_{i}, x^{t}\right) \\
& \underset{(N \rightarrow \infty)}{\approx} \sum_{t=0}^{T} \mathrm{~d} s^{t}\left\langle E C^{t}\left(\left.x\right|_{0} ^{t}, \omega\right), x^{t}\right\rangle \\
& \quad+\frac{1}{N_{s}} \sum_{s=1}^{N}\left(r _ { i } \sum _ { i = 0 } ^ { l } E \operatorname { m a x } \left\{0,\left\langle C^{t}\left(\left.x\right|_{0} ^{t}, \omega_{s}\right)\right.\right.\right. \\
& \left.\left.\quad-E C^{t}\left(\left.x\right|_{0} ^{t}, \omega\right)-\Delta \hat{c}_{i}, x^{t}\right)\right) \\
& B_{t} x^{t} \geqslant d^{t}, t=0,1, \ldots, T \\
& \sum_{k=0}^{t} R_{k}\left(\omega_{s}\right) x^{k}=r\left(\omega_{s}\right), t=0,1, \ldots, T \\
& \sum_{k=0}^{T} P_{k} x^{k} \leqslant \mathrm{e}^{t}, t=0,1, \ldots, T \\
& \quad 0 \leqslant x^{t} \leqslant \bar{x}^{t}, t=0,1, \ldots, T
\end{aligned}
$$

where $\left.x\right|_{0} ^{t}$ is $\left(x^{0}, x^{1}, \ldots, x^{t}\right) ; C^{t}\left(\left.x\right|_{0} ^{t}, \omega\right)$ are stochastic costs under condition that technology dynamics $\left.x\right|_{0} ^{t}$ is chosen and such that $C I_{t}=\left(C^{t}\left(\left.x\right|_{0} ^{t} 1, \omega\right) / C^{0}(\omega)\right)$, cost reduction index, has a distribution function described before (with number of doubling $N D_{t}$ calculated from $\left.x\right|_{o} ^{t}$ ) and initial distribution function for $C^{0}(\omega)$ is equal to $F_{0}() ; \Delta \hat{c}_{i}$ are given "threshold" values for total cost deviations and $R_{k}(\omega)$ and $r(\omega)$ reflect uncertain quantity-to-cost relations.

viewed as a sequential adaptation of a given initial energypolicy by learning from simulated history of its implementation.

In our test runs we used initially between 100 and 500 simultaneously drawn scenarios from each technology dynamics specification as an approximation of, in theory, infinite number of possible realizations. These ranges for the appropriate number of scenarios were obtained as a result of practical experiments and represent optimal trade-offs between exponentially growing computational complexity and reasonable accuracy of obtained solutions. Eventually, we decided that 250 simultaneously drawn scenarios are a sufficient number for a given technology dynamics. It is important to emphasize that it is not necessary to maintain high accuracy through many drawings during the initial calculation steps, when the value of the objective function is far from "optimal"differences between a solution value and the value of the new draw is much larger then errors due to "rough" stochastic approximation. However, at the final stage number of the drawing needs to be increased. At that stage, we use alternative drawing technique in order to get better estimates for error bounds.

\section{The solution technique and the computational approach}

As mentioned above, original stochastic global optimization non-smooth optimization is approximated by solving a sequence of large-scale linear optimization problems. It is done by applying a two-level nested structure. Global optimization part, which defines technological dynamics with respect to new unit installations for technologies with increasing returns to scale, is an implementation of adaptive global optimization random search algorithm specifically "tailored" to network flows optimization problems. (For description of such kind of algorithms see Horst and Pardalos, 1995; Pinter, 1996.) The inner algorithm is the interior-point method for linear optimization. The $P C x$ and $p P C x$ solvers were provided by the Argonne National Laboratory (Wright, 1996a, b; Czyzyk et al., 1997). These solvers are written in the $\mathrm{C}$ code, which we modified in order to increase computational efficiency for our specific problem formulation and to link them directly to the global optimization part.

One of the big advantages of the adaptive random search algorithm is that it does not require strictly sequential updating of approximated solution. Rather it refines the approximated solution at the time when information is available. This allowed us to devise a "parallel" adaptation of this technique. The inner linear optimization problem is relatively large and hard to solve. To find a solution for a given technology dynamics (with fixed uncertainty distribution parameters) by the global optimization algorithm requires approximately 10-40 min of CPU time on PC with Intel Pentium II 233 $\mathrm{MHz}$ and $128 \mathrm{MB}$ of RAM, depending on the number of simultaneous drawings from uncertain distributions, the number parameters to be considered and whether or not approximation of starting point is available (partial "hot" restart technique).

The original problem implementation was done on a CRAY T3E-900 supercomputer at the National Energy Research Scientific Computing Center (NERSC), in the US. NERSC is funded by the US Department of Energy, Office of Science, and is part of the Computing Sciences Directorate at the Lawrence Berkeley National Laboratory. All initial feasibility runs and a number of experiments were performed using 32 to 64 processing units on CRAY T3E-900. Later on, the problem was reported to IIASA computer network environment and 
re-implemented by using message passing interface (MPI) standard. We used public portable implementation of MPI - MPICH developed and supported by the Argonne National Laboratory and special implementation for Windows NT network clusters (WMPI) provided by the University of Coimbra, Portugal. Currently it is operational on a network cluster that contains from 6 to 16 Intel Pentium II $233 \mathrm{MHz}$ (as the number of PCs can be dynamically changed). Typical runs take from 22 to 46 wall clock hours. Due to extended logging procedure, calculations can be easily remotely operated, e.g., stopped and re-activated at any time. This technique allows utilization of computer "off-peak" and during the weekend hours.

\section{Major findings and the conclusion}

From the 520 alternative technology dynamics, about 53 resulted in scenarios with very similar overall energy systems cost. They all fall within $1 \%$ of the best values achieved. We designate this set of 53 technology dynamics as "optimal" because they are approximately equivalent with respect to the "optimality" criteria. Most of the statistical and other analyses here focus on these 53 optimal technology dynamics.

These 53 optimal, but fundamentally different, technology dynamics produce a wide range of alternative emergent energy systems. They all share the same useful energy demand trajectory but cover most of the range of carbon dioxide emissions found in the literature and unfold into all possible future energy systems structures. The underlying scenarios include futures that range from an increasing dependence on fossil energy sources to a complete transition to alternative energy sources and nuclear energy. Thus, one of the results of the analysis is that different structures of energy system emerge with similar overall costs, i.e., that there is a large diversity across alternative energy technology strategies. The strategies are path dependent and it is not possible to choose a priori "optimal" direction of energy systems development.

The scenarios from the literature span a wide range of future energy requirements and emissions due to the variation of the driving forces of future emissions such as the energy demand. In contrast, the set of scenarios with endogenous learning spans the range due to different technological dynamics alone. It is interesting to note that the "optimal" scenarios match quite closely the distribution of the scenarios from the literature but with a somewhat narrower range (they leave the extreme tails of the distribution uncovered). In contrast, the frequency distribution of the full set of 520 technology dynamics is different from the other two with many more scenarios in the mid-range of the distribution. This means that the optimal or most "cost-effective" development paths cor- respond quite closely to the scenario distribution from the literature. The "median" or "central" futures are underrepresented both in the literature and among the scenarios indicating that there appears to be a kind of "crowding-out" effect surrounding balanced and median type of scenarios. In any case, technological learning as specified in our approach leads to future energy systems that are marked either by high or low emissions ranges (with one single useful demand trajectory) demonstrating a kind of implicit bifurcation across the range of possible emissions.

Another result of the analysis is that the endogenous technology learning with uncertainty and spillover effects has the greatest impact on the emerging structures of energy system during the first few decades of the next century. Over these "intermediate" periods of time these two processes create effective lock-in effects and increasing returns to adoption. In the very long run, however, all of these effects are not of great importance. The reason is that over such long periods many doublings of capacity of all technologies with inherent leaning occur so that there are few relative cost advantages that result from large investments in some technologies and clusters. Therefore, the main finding is that under uncertainty the near-term investment decisions in new technologies are more important in deciding the direction of long-term development of the energy system than are decisions that are made later, toward the end of the time horizon. Thus, the most dynamic phase in the development of future energy systems will occur during the next few decades. It is during this period that there is a high freedom of choice across future technologies and many of these choices would lead to high spillover learning effects for related technologies.

One policy implication that can be made based on the emerging dynamics and different directions of energy systems development in this analysis is that future RD\&D efforts and investments in new technologies should be distributed across "related" technologies rather than be directed at only one technology from the cluster even if it appears as a "winner." Another implication is that it is better not to spread RD\&D efforts and technology investments across a large portfolio of future technologies. It is rather better to focus on (related) technologies that might form technology clusters.

\section{Acknowledgements}

We would like to thank Sabine Messner, Gordon MacDonald, Yuri Ermoliev and Manfred Strubegger, all from IIASA, for their help and advise. Sabine Messner and Gordon MacDonald worked with us on the grant from the National Energy Research Scientific Computing Center at Lawrence Berkeley National Laboratory that is funded by the US Department of Energy. This grant 
allowed the original problem implementation on a GRAY T3E-900 supercomputer and we are grateful for the financial support provided by the US Department of Energy. We also gratefully acknowledge that the original MESSAGE model implementation and technology assumptions are based on the work of Sabine Messner. We are also grateful to Yuri Ermoliev, who helped with the development of the solution methods and for his continuous help and advise. Last, but not least, we thank Manfred Strubegger, who has provided the fossil energy supply functions, has implemented important changes in the problem solution processing module and has developed a new script we used for storing the results of this analysis.

We would also like to thank colleagues from other institutions, who have provided software and support for our research. They include Michael Wagner and Steve Wright from the Argonne National Laboratory and Cornell University, respectively, and Francesca Verdier from the National Energy Research Scientific Computing Center at Lawrence Berkeley National Laboratory for her assistance and help.

\section{References}

Bankes, S.C., 1993. Exploratory Modeling and Policy Analysis. RAND/RP-211, Santa Monica, U.S.A.

Christiansson, L., 1995. Diffusion and Learning Curves of Renewable Energy Technologies, WP-95-126, International Institute for Applied Systems Analysis, Laxenburg, Austria.

Czyzyk, J., Mehrotra, S., Wagner, M., Wright, S., 1997. PCx user guide (Version 1.1). Technical Report, OTC 96/01. (Available online at http://www-unix.mcs.anl.gov/otc/Tools/PCx/doc/PCx-user.ps).

Ermoliev, Y., Wets, R.J.-B., 1988. Numerical Techniques for Stochastic Optimization. Springer, Berlin.

Ermoliev, Y., Norkin, V., 1995. On Nonsmooth Problems of Stochastic Systems Optimization. WP-95-096. IIASA, Laxenburg, Austria.

Ermoliev, Y., Norkin, V., 1998. Monte Carlo Optimization and Path Dependent Nonstationary Laws of Large Numbers. IR-98-009. IIASA, Laxenburg, Austria.

Foray, D., Grübler, A., 1990. Morphological analysis, diffusion and lock-out of technologies: ferrous casting in France and Germany. Research Policy 19(6), 535-550.

Golodnikov, A., Gritsevskyi, A., and Messner, S., 1995. A Stochastic Version of the Dynamic Linear Programming Model MESSAGE III. WP-95-94. IIASA Laxenburg, Austria.

Grübler, A., 1998. Technology and Global Change. Cambridge University Press, Cambridge.

Grübler, A., Gritsevskyi, A., 2000. A model of endogenized technological change through uncertain returns on learning. Ukranian Economic Review, VII (in press).
Grübler, A., Messner, S., 1996. Technological uncertainty. In: Nakienovi, N., Nordhaus, W.D., Richels, R., Toth, F. (Eds.), Climate Change: Integrating Science, Economics and Policy. CP-96-1, IIASA, Laxenburg, Austria.

Horst, R., Pardalos, P.M., (Eds.), 1995. Handbook of Global Optimization. Kluwer, Dordrecht.

Lempert, R.J., Schlesinger, M.E., Bankes, S.C., 1996. When we don't know the costs or the benefits: adaptive strategies for abating climate change. Climatic Change, 33.

MacGregor, P.R., Maslak, C.E., Stoll, H.G., 1991. The Market Outlook for Integrated Gasification Combined Cycle Technology, General Electric Company, New York, NY, USA.

Markowits, H., 1959. Portfolio Selection. Wiley, New York.

Messner, S., 1995. Endogenized Technological Learning in an Energy Systems Model. WP-95-114. IIASA, Laxenburg, Austria.

Messner, S., Golodnikov, A., Gritsevskii, A., 1996. A stochastic version of the dynamic linear programming model MESSAGE III. Energy 21(9).

Messner, S., Strubegger, M., 1991. Part A: User's Guide to CO2DB: The IIASA $\mathrm{CO}_{2}$ Technology Data Bank - Version 1.0. WP-91-069. IIASA, Laxenburg, Austria.

Morita, T., Lee, H.-C., 1998. Apendix to emissions scenarios database and review of scenarios. Mitigation and Adaptation Strategies for Global Change 3(2-4), 121-131.

Nakićenović, N., Grübler, A., Ishitani, H., Johansson, T., Marland, G., Moreira, J.R., Rogner, H.-H., 1996. Energy primer. In: Climate Change 1995: Impacts, Adaptations and Mitigation of Climate Change: Scientific-Technical Analysis, Contribution of Working Group II to the Second Assessment Report of the IPCC. Cambridge University Press, Cambridge, UK, pp. 75-92.

Nakićenović, N., 1996. Technological change and Learning. In: Nakicenovi, N., Nordhaus, W.D., Richels, R., Toth, F. (eds), Climate Change: Integrating Science, Economics and Policy. CP-96-1, IIASA, Laxenburg, Austria.

Nakićenović, N., 1997. Technological change as a learning process. Paper presented on Technological Meeting'97, IIASA, Austria.

Nakićenović, N., Grübler, A., McDonld, A. (eds), 1998a, Global Energy Perspectives, Cambridge University Press, Cambridge, UK.

Nakićenović N., Victor, N., Morita, T., 1998b. Emissions scenarios database and review of scenarios. Mitigation and Adaptation Strategies for Global Change 3(2-4), 95-120.

Pinter, J., 1996. Global Optimization in Action. Kluwer, Dordrecht.

Robalino, D., Lempert, R., 2000. Carrots and sticks for new technclogy: Crafting greenhouse gas reduction policies for a heterogeneous and uncertain world. Integrated Assessment 1(1), 1-19.

Rogner, H., 1997. An assessment of world hydrocarbon resources. Annual Review of Energy and the Environment 22, 217-262.

Strubegger, M., Reitgruber, I., 1995. Statistical Analysis of Investment Costs for Power Generation Technologies. WP-95-109. IIASA, Laxenburg, Austria.

Watanabe, C., 1995. Identification of the role of renewable energy. Renewable Energy 6(3), 237-274.

Wright, S.J., 1996a. Primal-Dual Interior-Point Methods. SIAM, London, UK.

Wright, S.J., 1996b. Modified cholesky factorizations in interior-point algorithms for linear programming. Preprint ANL/MCS-P6000596. Argonne National Laboratory, U.S.A. 
